\title{
Affecting an Ultra-High Work Function of Silver
}

Jin He, ${ }^{[\mathrm{a}]}$ Jeff Armstrong, ${ }^{[\mathrm{b}]}$ Peixi Cong, ${ }^{[\mathrm{c}],[\mathrm{d}]}$ Barak Menagen, ${ }^{[\mathrm{a}]}$ Lior Igaher, ${ }^{[\mathrm{a}]}$ Andrew M. Beale, ${ }^{[\mathrm{c}],[\mathrm{d}]}$ Lioz Etgar $^{[\mathrm{a}]}$ and David Avnir ${ }^{[\mathrm{a}], *}$

[a] Dr. Jin He, Barak Menagen, Lior Igaher, Prof. Dr. Lioz Etgar, Prof. Dr. David Avnir Institute of Chemistry and The Center for Nanoscience and Nanotechnology

The Hebrew University of Jerusalem

Jerusalem 9190401, Israel

E-mail: david.avnir@mail.huji.ac.il

[b] Dr. Jeff Armstrong

ISIS Facility

Rutherford Appleton Laboratory

Harwell Oxford, Didcot, Oxfordshire OX11 0QX, UK

[c] Peixi Cong, Prof. Dr. Andrew M. Beale

Department of Chemistry

University College of London

Gordon Street, London WC1H 0AJ, UK

[d] Peixi Cong, Prof. Dr. Andrew M. Beale

Research Complex at Harwell

Rutherford Appleton Laboratory

Harwell Oxford, Didcot, Oxfordshire OX11 OFA, UK 


\begin{abstract}
Efficient control of the work function (WF) of metals and their increase to ultra-high values are crucial for their applications in functional devices applying interfacial charge transport processes. We report an ultra-high increase in the WF of silver, from 4.26 to $7.42 \mathrm{eV}$, that is, an increase of up to $\sim 3.1 \mathrm{eV}$. This, apparently, highest WF increase on record for metals and is supported by recent computational studies which predict the potential ability to affect an increase of the WF of metals by more than $4 \mathrm{eV}$. We achieved the ultra-high increase by a new approach: Rather than using the common method of 2D adsorption of polar molecules layers on the metal surface, we have incorporated WF modifying components - L-cysteine and $\mathrm{Zn}(\mathrm{OH})_{2}$ - within the metal, resulting in a 3D architecture. Detailed material characterization by a large array of analytical methods was carried out (XRD, SEM, EDS mapping, TGA/MS, synchrotron X-ray absorption, inelastic neutron scattering, Raman spectroscopy), the combination of which points to a WF enhancement mechanism which is based on affecting directly the charge transfer ability of the metal separately by cysteine and hydrolyzed zinc(II), and synergistically by the combination of the two components through the known $\mathrm{Zn}$ cysteine finger redox trap effect. Some additional properties include the ability to finetune the WF from the pure silver values and up; the conductivity of the doped silver remains practically unaffected; the WF is stable beyond 3 months of storage; and it is heat resistant up to $150^{\circ} \mathrm{C}$. The ability to tailor WF changes from the standard value of silver and up over a wide range, will certainly find its applications wherever tuning of the WF is needed for the design of charge transport devices.
\end{abstract}

Key words: work function; ultra-high; doping metals; silver; Kelvin probe 


\section{Introduction}

The work function (WF) of a solid is the energy necessary to remove an electron originally at the Fermi level and place it at rest just outside the surface. ${ }^{1}$ This property affects major applications of metals which involve charge transport across their interfaces, including organic electronic devices, thermionic electron guns, sensing microelectronics and more. ${ }^{2-4}$ Methods for fine-tuning the WF in order to tailor it for the specific applications have been developed. By-far, the most common method used for modifying this property in metals has been the adsorption or covalent binding of polar molecules layers on the metal surface, thus creating an interfacial dipole barrier for the charge transport. ${ }^{5-7}$ Beyond fine-tuning, a long standing challenge has been to increase the WF as high as possible, in order to further expand the range of possibilities of tailoring the desired WF value. Recent computational work predicted that the WF change of $\mathrm{Ag}$ or Au by a chemical modification can be as much as $4 \mathrm{eV}$ and above. ${ }^{8,9}$ Yet, to the best of our knowledge, the highest experimental increase of metal's WF did not exceed $\sim 2 \mathrm{eV}$, as reported, for instance, by de Boer, ${ }^{5}$ Hofmann, ${ }^{10}$ and Frisbie. ${ }^{7}$

Recently we have introduced an entirely new methodology for affecting the WF of metals, namely the doping of the metal with WF-modifying agents; that is, we move from derivatizing the $2 \mathrm{D}$ interface to the $3 \mathrm{D}$ volume of the metal. With this molecular doping we demonstrated the possibility to fine-tune the WF of gold and silver within the modest range of up to $1 \mathrm{eV} .{ }^{11}$ Being a new approach and following the above cited theoretical predictions, we set to explore the possibility to utilize the doping methodology in order to push the WF of silver into the ultra-high domain, which in the literature is defined as an increase of $2-3 \mathrm{eV} \cdot{ }^{9-11}$ The materials methodology of molecular doping of metals employed here involves a variety of reducing processes of metal cations in the presence of the molecule or nanoparticle (NP) to be entrapped (see ref.'s ${ }^{12,13}$ for reviews and ${ }^{11,14-18}$ for recent examples). The resulting material is the metal in the form of tightly agglomerated nanometric crystals, incorporating the dopant molecule or NPs. The dopant is firmly held by the physical caging and by interactions between its functional moieties and the metallic surface of the nanocrystals that form the cages. It has been repeatedly shown in previous applications studies of this materials methodology that this 3D architecture is completely different from regular 2D adsorption, and various specific applications, such as in catalysis, ${ }^{17}$ biomaterials, ${ }^{15,16}$ fuel cell, ${ }^{14}$ and more, ${ }^{18}$ are achieved only with the $3 \mathrm{D}$ entrapment. This is so because unlike $2 \mathrm{D}$ adsorption where the molecules interact with the metal typically through one 
moiety, in 3D entrapment the interaction between the dopant and the molecule is through all of its parts - therefore the effect of the electrons ocean of the metal on the dopant, and of the dopants orbitals on the metal, is different and by far stronger.

We report now that the goal of pushing the WF of silver closer to the higher values predicted theoretically, was achieved: An ultrahigh increase in the WF of silver - from 4.26 to $7.42 \mathrm{eV}$ - that is, an increase of up to $\sim 3.1 \mathrm{eV}(71.5 \mathrm{kcal} / \mathrm{mole})$, was obtained, apparently the highest on record for metals. The experimental approach has been a two-components doping of silver - L-cysteine and zinc hydroxide nanocrystals each affecting the WF by the surface dipole formed at the interface, as well as by a mutual synergistic mechanism, all of which are detailed below. The effect is tunable, allowing one to fine-tune the WF values of silver, over the wide range of $3.1 \mathrm{eV}$, as desired. Here are the details:

\section{Results and discussion}

\section{The chemistry of the entrapment:}

To achieve the dual-entrapment in silver, the two dopants - (see Experimental Details in the Supporting Information (SI), and Scheme S1 in the SI), several processes and reactions between $\mathrm{AgNO}_{3}$, zinc and L-cysteine, take place. Eq. (1) represents the formation of metallic silver atoms, which grow to nanocrystal nuclei of Ag, and which then grow further to the entrapping aggregated nanocrystals of metallic silver.

$$
\mathrm{Zn}+\mathrm{AgNO}_{3} \rightarrow \mathrm{Ag}+\mathrm{Zn}\left(\mathrm{NO}_{3}\right)_{2}
$$

Zinc was selected as a reducing agent because it proved to be efficient in previous molecular doping of metals studies. ${ }^{17,19}$ Zinc nitrate is formed as a by-product, most of which is washed away, but some of which enters reaction (3) described below. A small excess of $\mathrm{Zn}$ is taken in order to produce also the oxidative hydrolysis product, $\mathrm{Zn}(\mathrm{OH})_{2}$, as a co-dopant according to

$$
\mathrm{Zn}+2 \mathrm{H}_{2} \mathrm{O} \rightarrow \mathrm{Zn}(\mathrm{OH})_{2}+\mathrm{H}_{2} .
$$

Under the reaction conditions, $\mathrm{Zn}\left(\mathrm{NO}_{3}\right)_{2}$ is not hydrolyzed to $\mathrm{Zn}(\mathrm{OH})_{2}$. Of the two reduction reactions, (1) and (2), the reduction by zinc of the silver cation (reduction potential of $-0.76 \mathrm{~V})$ is much faster than the reduction of water protons $(0.0 \mathrm{~V})$, and this difference leads to the ability of incorporating a small amount of $\mathrm{Zn}(\mathrm{OH})_{2}$ nanocrystals within the forming silver matrix. $\mathrm{Zn}\left(\mathrm{NO}_{3}\right)_{2}$ reacts with $\mathrm{Zn}(\mathrm{OH})_{2}$ forming zinc hydroxide nitrate: ${ }^{20}$

$$
\text { (3) } \mathrm{Zn}\left(\mathrm{NO}_{3}\right)_{2}+4 \mathrm{Zn}(\mathrm{OH})_{2}+2 \mathrm{H}_{2} \mathrm{O} \rightarrow \mathrm{Zn}_{5}(\mathrm{OH})_{8}\left(\mathrm{NO}_{3}\right)_{2}\left(\mathrm{H}_{2} \mathrm{O}\right)_{2} \text {, }
$$


but as we shall see below, the addition of cysteine, quenches this reaction, apparently through the protective interaction of cysteine with $\mathrm{Zn}(\mathrm{OH})_{2}:{ }^{21}$

$$
\mathrm{Cys}-\mathrm{SH}+\mathrm{Zn}(\mathrm{OH})_{2} \rightarrow\left(\mathrm{Cys}^{-} \mathrm{S}_{2}\right)_{2} \mathrm{Zn}^{2+}+\mathrm{H}_{2} \mathrm{O} \text {. }
$$

The formation of the mercaptide $\left(\mathrm{Cys}-\mathrm{S}^{-}\right)_{2} \mathrm{Zn}^{2+}$ is a key process in the biochemistry of Zn-metalloproteins, ${ }^{22}$ known as the "zinc-finger", ${ }^{23,24}$ and of relevance to our report is its function as a powerful redox pair for electron trapping. ${ }^{25,26}$ The cysteine mercaptide of silver ${ }^{27}$ appears in the process as a transient gel that, however, does not interfere with the reduction process, because $\mathrm{Zn}$ is a strong enough reducing agent to operate on that silver compound as well.

An important interaction to be considered as well for the entrapment process and for the mechanism of the WF increase, is the well-known interaction of thiol groups with d-element metallic surfaces, ${ }^{28-30}$ occurring in our case between Cys-SH and both the metallic zinc and the formed metallic silver. ${ }^{31}$ This interaction facilitates the homogeneous distribution of both dopants, and contributing to it are also the amino and the carboxylate group which are good adsorptive anchors centers to a metal. ${ }^{32}$ At the low concentrations of cysteine we use, it is not blocking the $\mathrm{Zn}$ from reducing the $\mathrm{Ag}^{+}$. Thus, while Gibbs free energy of metallic zinc-cysteine formation is $-25 \mathrm{~kJ} \mathrm{~mol}^{-1}$, the free energy of the reduction of $\mathrm{Ag}^{+}$by zinc is $-308 \mathrm{~kJ} \mathrm{~mol}^{-1} .^{21}$

What then are the reaction conditions which result in a doped metal? This question has been treated in detail in previous studies of molecular entrapment within metals,${ }^{16,17}$ where, in brief, the following mechanism has been suggested: Shortly after mixing the metal salt, the reducing agent and the dopants, reversible dopant-metal cation interactions occur (such as forming silver cysteinate), and the reducing agent is operating on both the free and the complexed cations. As a result, metallic atoms form, which begin to aggregate into metal nanocrystals, the elementary building blocks of the final doped metal. The dopant interacts reversibly with these forming crystallites, while more metal continues to form. If the condition that the residence time of the adsorbed dopant is longer than the rate of reduction and formation of more metal is fulfilled, then precipitation/aggregation of the metal will capture the dopant in 3D metallic cages. In our case this condition is fulfilled, since, as mentioned above, cysteine strongly chemisorbs on metals, and this occurs during a fast reduction reaction. As the reduction - reaction (1) - proceeds, its rate slows down, and the slower hydrolysis - reaction (2) - becomes relatively more pronounced. At a later stage of the reduction reaction, the small excess of $\mathrm{Zn}$ has already shrunk to very small nanoparticles, accelerating the 
hydrolysis to $\mathrm{Zn}(\mathrm{OH})_{2}$ nanoparticles (and also to $\mathrm{Zn}$ nanoparticles coated with $\left.\mathrm{Zn}(\mathrm{OH})_{2}\right)$. We denote the hydrolysis product of metallic zinc as $\mathbf{Z n}(\mathbf{I I})$ and resulting doubly doped silver as cys/Zn(II)@Ag.

\section{The work function observations:}

Table 1 displays the work functions and resistivities of cys/Zn(II)@Ag discs of various compositions, along with comparisons to pure Ag, Zn(II)@ Ag and cys@ Ag discs. The WF of the pure silver prepared by the same procedure but without the dopants is $4.26 \mathrm{eV}$; the WF of the dual doped silver is higher by more than $3 \mathrm{eV}-7.42 \mathrm{eV}$ - at a starting molar ratio of 0.1:0.6:1.0 cysteine: $\mathrm{Zn:Ag}$. 2D maps of one of the cys/Zn(II)@Ag compositions and one of the blanks (cys@ Ag) are shown in Figure 1, and reveal a homogenous distribution of this property on a $2 \times 2 \mathrm{~mm}$ scale. Other compositions are shown in the Table 1, and it is remarkable that even $2 \%$ mol doping of zinc increases the WF by $1.5 \mathrm{eV}$ to $5.76 \mathrm{eV}$. Removing $\mathrm{Zn}$ all together, that is, having only cysteine doping, results in an increase to $5.05 \mathrm{eV}$, a value which in agreement with the fine-tuning values of WF we reported for other organic molecules molecular doping of metals. ${ }^{8}$ Using only excess $\mathrm{Zn}(0.6: 1, \mathrm{Zn}: \mathrm{Ag})$ the WF value is again moderately changed to $5.36 \pm 0.15 \mathrm{eV}$. Thus, combining the two dopants shows that there is strong synergism of action between them.

Several additional important observations were made: First, the conductivity of the doped discs remains high, only marginally smaller than that of the pure metal (Table 1); second, and the WFs measured were stable for at least 3 months; and third, the high WF values are thermally stable, up to the decomposition temperature of cysteine (150 ${ }^{\circ} \mathrm{C}$ ) - see TGA analysis below for details. Next we move to a detailed characterization of the doped silver, which we use for a proposed mechanism of the WF strong enhancement. 
Table 1 Work functions and resistivities for cysteine/Zn(II)@ Ag and reference samples

\begin{tabular}{llll}
\hline Sample & $\begin{array}{l}\text { Cysteine:Zn:Ag } \\
\text { Starting molar ratio }\end{array}$ & WF $(\mathbf{e V})$ & Resistivity $(\mathbf{\Omega} * \mathbf{c m})$ \\
\hline $\mathbf{1 ~ A g}$ & & $\mathbf{4 . 2 6} \pm \mathbf{0 . 1 0}$ & $3.0 \pm 0.2 \times 10^{-6}$ \\
\hline $\mathbf{2}$ cys/Zn(II)@ Ag & $0.10: 0.60: 1.00$ & $\mathbf{7 . 4 2} \pm \mathbf{0 . 1 8}$ & $4.8 \pm 0.3 \times 10^{-6}$ \\
\hline 3 cys/Zn(II)@Ag & $0.10: 0.55: 1.00$ & $6.50 \pm 0.25$ & $4.5 \pm 0.3 \times 10^{-6}$ \\
\hline 4 cys/Zn(II)@ Ag & $0.10: 0.52: 1.00$ & $5.76 \pm 0.15$ & $4.1 \pm 0.4 \times 10^{-6}$ \\
\hline 5 cys@ Ag & $0.10: 1.00$ & $5.05 \pm 0.09$ & $3.9 \pm 0.2 \times 10^{-6}$ \\
\hline $6 \mathrm{Zn}(\mathrm{II}) @ \mathrm{Ag}$ & $0.60: 1.00$ & $4.86 \pm 0.15$ & $5.5 \pm 0.3 \times 10^{-5}$ \\
\hline $7 \mathrm{Zn}(\mathrm{II}) @ \mathrm{Ag}$ & $1.00: 1.00$ & $5.06 \pm 0.20$ & $3.2 \pm 0.3 \times 10^{-2}$ \\
\hline
\end{tabular}

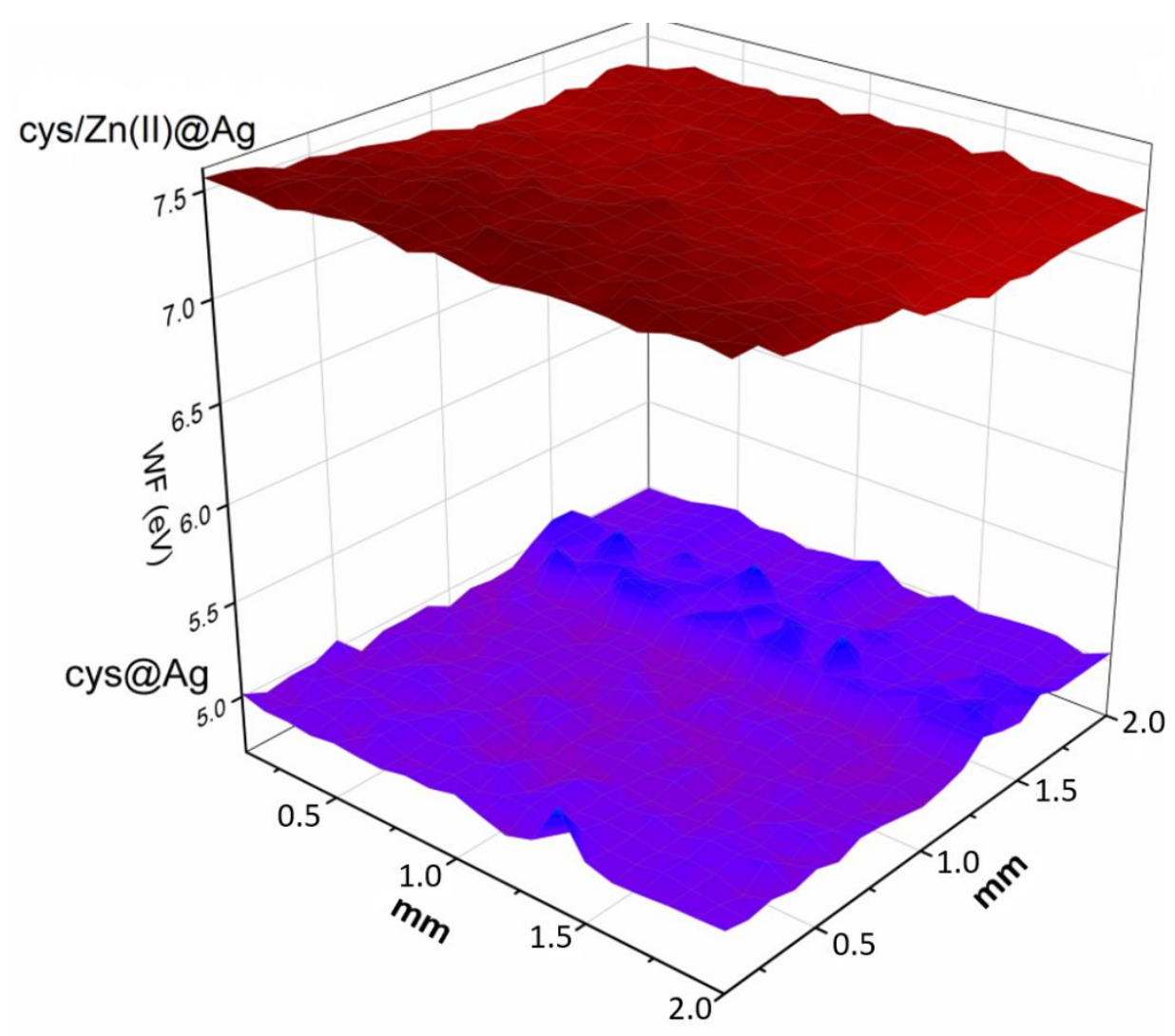

Figure 1. Work function 2D mapping of cys/Zn(II)@ Ag at the maximal WF effect ratio (cysteine:Zn:Ag = 0.1:0.6:1.0, starting molar ratio), compared with the WF map of cys@Ag for reference (cysteine:Zn:Ag=0.1:0.5:1.0, starting molar ratio). 


\section{Material characterization:}

The composition for which the ultra-highest WF value $-7.42 \pm 0.18 \mathrm{eV}$ (Table 1) - was obtained, is at a molar ratio of cys: $\mathrm{Zn:Ag}$ of 0.10:0.10:1.00, (that is, an intial synthetic molar ratio of 0.10:0.60:1.00). The results of the XRD analysis for cys/Zn(II)@ Ag prepared with increasing initial zinc amounts from 0.55 to 1 at a fixed cysteine/Ag molar ratio of 0.1:1 are shown in Figure 2A. It is clearly seen, even with a molar $\mathrm{Zn}$ amount of 0.7 (40\% excess for the reduction), that the dominant peaks are of FCC metallic silver, appearing at $2 \theta=38.1,44.4,64.5,77.4$ and $81.6^{\circ}$ which are associated with the (1 11), (2 00), (2 20), (3 11) and (2 22) crystallographic planes, respectively. Applying Scherrer's equation on the peaks of the XRD spectra indicate that the average size of the elementary nanocrystals building blocks is around $35 \mathrm{~nm}$. This dominant pure silver phase indicates a quite uniform doping of the cysteine and of the excess $\mathrm{Zn}$ and it also indicates that the $\mathrm{Zn}$ (II) particles are nanometric or even subnanometric particles. In fact, it is possible to see some $\mathrm{Zn}(\mathrm{OH})_{2}$ phase in Figure 2A only if a large excess of $\mathrm{Zn}$ - cys: $\mathrm{Zn}: \mathrm{Ag}$ of 0.10:1.00:1.00 (starting molar ratio) - is taken (Figure 2A, green line). Interestingly, $\mathrm{Zn}_{5}(\mathrm{OH})_{8}\left(\mathrm{NO}_{3}\right)_{2}\left(\mathrm{H}_{2} \mathrm{O}\right)_{2}$ (eq. 3) is seen only if cysteine is removed (at $2 \theta=9.1$ and $18.1^{\circ},{ }^{33}$ Figure $\mathbf{2 A}$, purple line), but only as a trace in its presence (Figure $\mathbf{2 A}$, green line). This proves the binding of cysteine to $\mathrm{Zn}(\mathrm{OH})_{2}$, which is important for the mechanism of the WF increase given below.

X-ray absorption fine structure (XAFS) allows one to determine the chemical environment (valence states, coordination number) as well as short range structure around the silver and zinc atoms upon doping. The silver K-edge XAFS data is shown in Figure 2B, S2, S3 and S4A. Both the X-ray absorption near-edge structure (XANES, Figure 2B) and the k3-weighted X-ray absorption fine structure spectra (EXAFS) (Figure S2) closely match that of the bulk Ag (metal foil), indicating that all the $\mathrm{Ag}$ is in the metallic phase. This is also demonstrated by the Fourier transformed EXAFS (FT-EXAFS) shown in Figure S4A. Furthermore, the FT-EXAFS spectra demonstrate a noticeable decrease in intensity of the $\mathrm{Ag}-\mathrm{Ag}$ shell of the doped silver, which is expected when foreign species are incorporated. The spectra profiles of pure Ag and cysteine adsorbed on $\mathrm{Ag}$ are virtually identical, while a decrease in the $\mathrm{Ag}-\mathrm{Ag}$ scattering intensity is observed in the spectra of the doped materials, cys@Ag and cys/Zn(II)@Ag, which again demonstrates the inherent difference between 2D adsorption and the 3D entrapment. This decrease is even more pronounced in 
cys/Zn(II)@Ag, which indicates an enhanced co-doping effect on the silver. Evidence of $\mathrm{Ag}-\mathrm{S}$ bond is seen in the Ag FT-EXAFS moduli of cys@ $\mathrm{Ag}$ and cys/Zn(II)@ $\mathrm{Ag}$ (Figure S4A and Table S1. Detailed analysis appears in the SI). The Zn K-edge XANES spectra (Figure 2C) clearly show that the rise edge of cys/Zn(II) @ Ag is 4.2 $\mathrm{eV}$ higher in energy compare to the reference metallic $\mathrm{Zn}$ foil which indicates that the $2+$ oxidation state of $\mathrm{Zn}$ is the dominant species. ${ }^{34}$ The Zn FT-EXAFS spectra (Figure S4B) reveal also that coordination environments are identical to $\mathrm{Zn}-\mathrm{O}$ bond, which proves that the entrapped $\mathrm{Zn}$ is in the hydrolyzed form. The second shell scattering around $3.35 \AA$ in the Zn FT-EXAFS spectrum (Figure S4B) reveals the scattering from a heavier atom - $\mathrm{Ag}$ - which indicates the existence of the $\mathrm{Zn}(\mathrm{OH})_{2} / \mathrm{Ag}$ interface. This is relevant to the charge transfer between the two components as part of the mechanism of the WF increase (below). More XAFS analyses are provided and discussed in Figures S2, S3, S4 and Table S1, SI. 

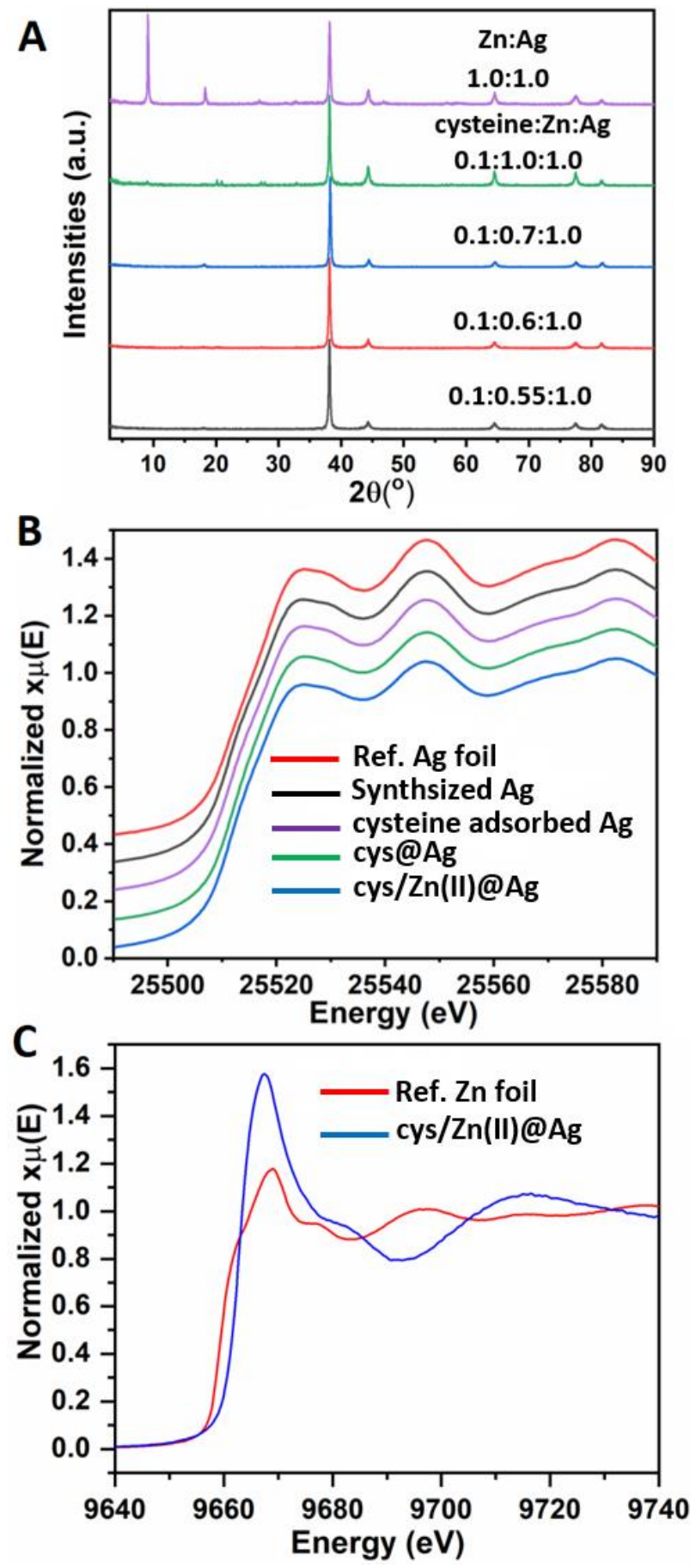

Figure 2. A) XRD patterns of cys/Zn(II)@ Ag prepared with different molar ratios of zinc and comparison with the absence of cysteine (shown are the starting molar ratios); B) Normalized Ag K-edge XANES spectra of (from top to bottom) Ag metal foil, synthesized Ag powder, cysteine adsorbed on Ag, cys@ $\mathrm{Ag}$ and cys/Zn(II)@ Ag (0.1:0.6:1.0, starting molar ratio); C) Normalized Zn K-edge XANES spectra of Zn metal foil and cys/Zn(II)@ $\mathrm{Ag}$ (0.1:0.6:1.0, starting molar ratio). 


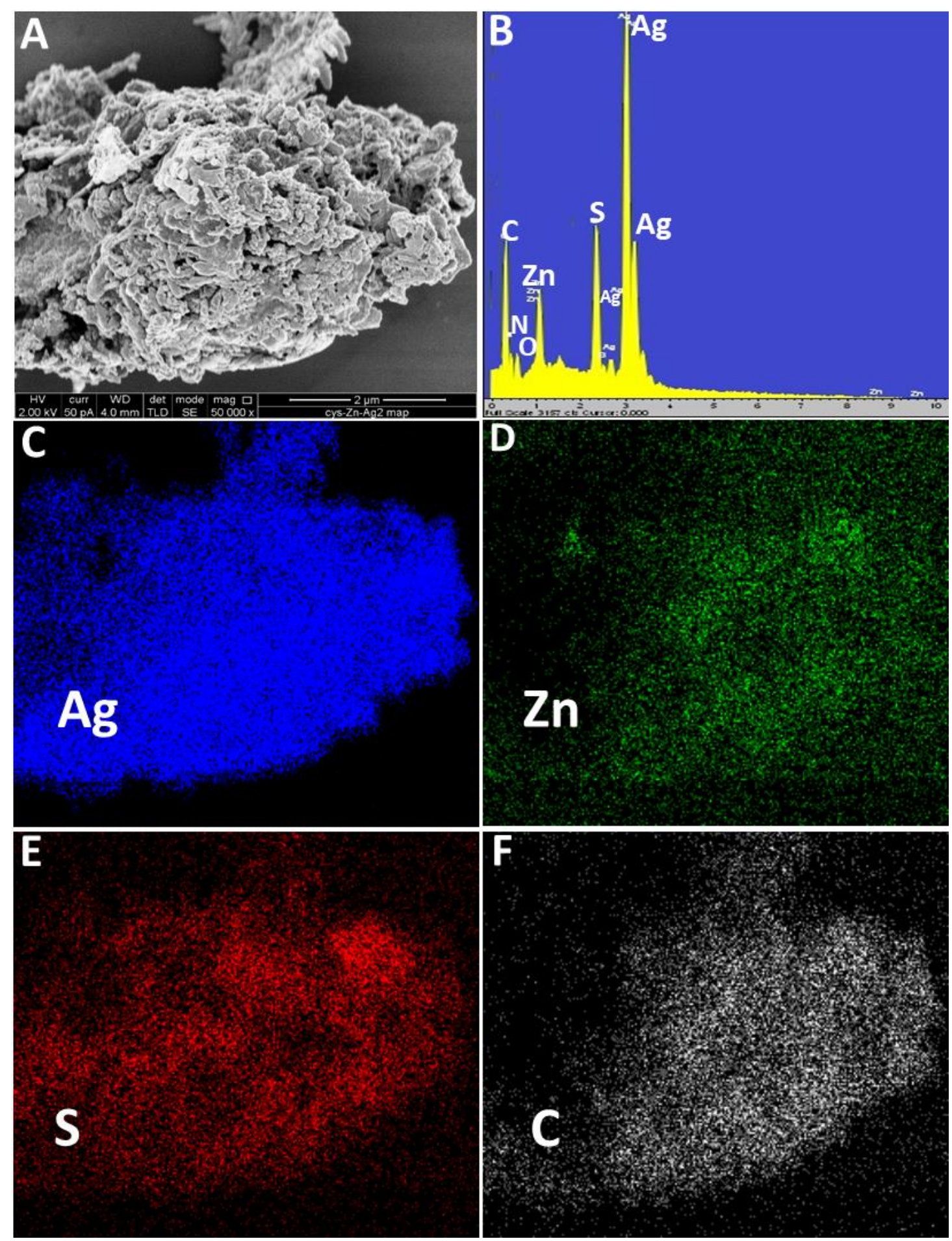

Figure 3. Cys/Zn(II)@ Ag (0.1:0.6:1.0, starting molar ratios): A) SEM image, scale bar $=2 \mu \mathrm{m}$; B) EDAX spectrum. EDS maps: C) Ag; D) Zn; E) S and F) C.

A typical high-resolution scanning electron microscopy (HR-SEM image) of the cys/Zn(II)@Ag composite (cysteine/Zn/Ag=0.1:0.6:1.0, the maximal WF increase composition, Table 1) is displayed in Figure 3A. It is seen that the composite is made of hierarchically aggregated nanometric crystallites. This microstructure leads to a 
nitrogen-BET (Brunauer-Emmett-Teller) surface area of $6.21 \mathrm{~m}^{2} \mathrm{~g}^{-1}$ displaying an adsorption-desorption isotherm typical of interstitial porosity (Figure S5) with a Barrett-Joyner-Halenda $(\mathrm{BJH})$ average mesopore diameter of $3.6 \mathrm{~nm}$. After being pressed into disc for WF measurement, the mesoporous structure collapsed into almost non-porous materials (surface area- $0.65 \mathrm{~m}^{2} \mathrm{~g}^{-1}$ ). Coupling energy dispersive X-ray (EDAX) analysis (Figure 3B) with SEM imaging reveals the triple organic-inorganic hybrid nature of the composite with the appearance of silver, zinc, oxygen, carbon, nitrogen and sulfur. Mapping of the elements (Figure 3C-F) demonstrates the homogeneous nature of the triple composite, at least on the scale of the diameter of the beam of electrons $(\sim 1 \mu \mathrm{m})$.
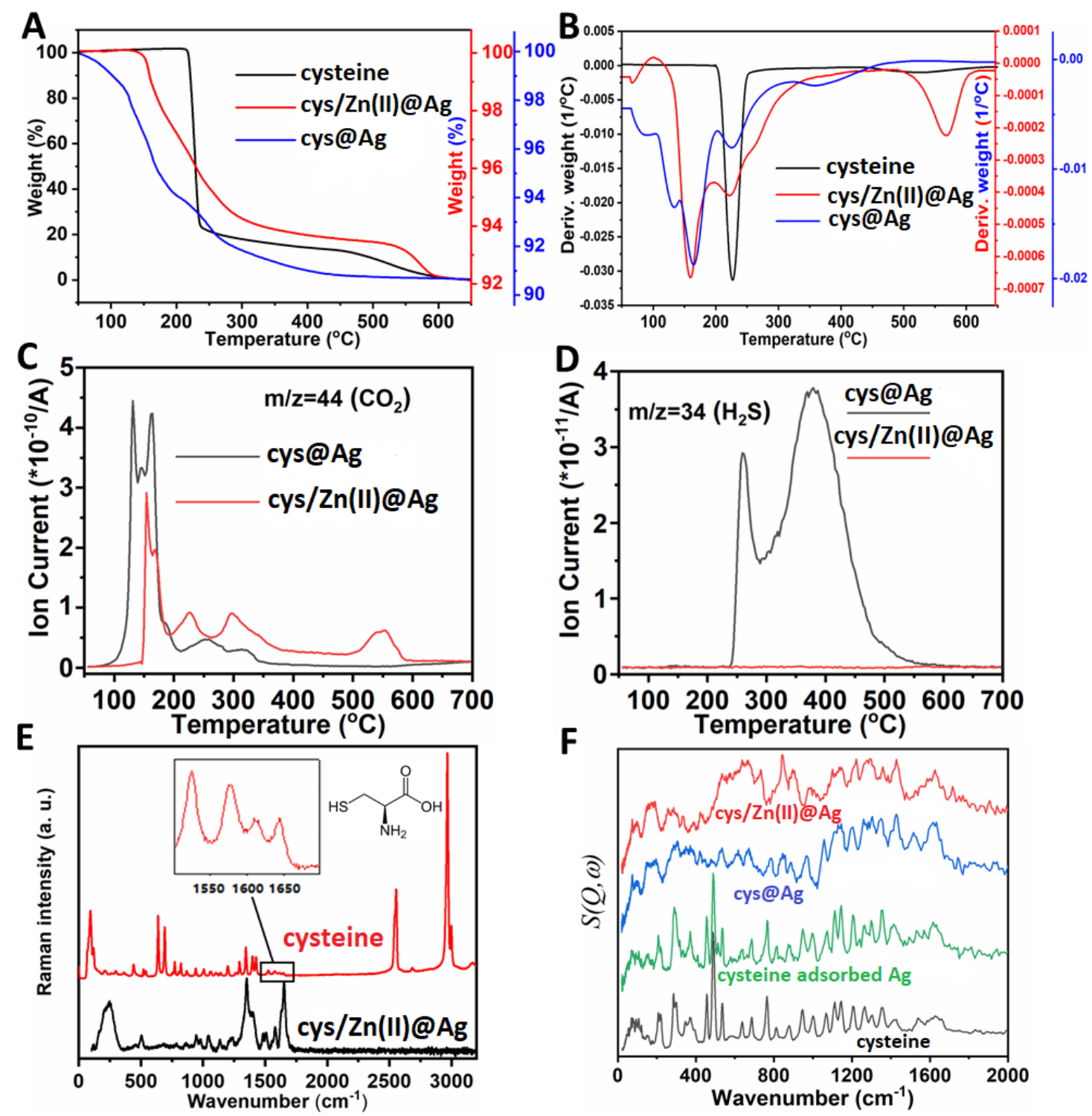

Figure 4. A) Thermogravimetric analysis (TGA) of the weight loss of cysteine(/Zn(II))@Ag (starting molar of 0.1:(0.6):1.0); B) the weight loss first 
derivative graphs and mass spectrometry coupled to TGA; C) $\mathrm{m} / \mathrm{z}=44\left(\mathrm{CO}_{2}\right)$; D) $\mathrm{m} / \mathrm{z}=34\left(\mathrm{H}_{2} \mathrm{~S}\right)$; E) Raman spectra of cysteine and cys/Zn(II)@ Ag (0.1:0.6:1.0, starting molar ratio) and F) INS spectra of (from top to bottom) cys/Zn(II)@ Ag (0.1:0.6:1.0, starting molar ratio), cys@ Ag, cysteine adsorbed on Ag and cysteine.

Thermogravimetric analysis (TGA) of cys/Zn(II)@ Ag focuses on the organic component (cysteine), and it is compared in Figure 4A-D to pure cysteine and to cys@ Ag (without co-entrapped Zn). It is seen that the entrapment of cysteine, in either $\mathrm{Ag}$ or in $\mathrm{Zn}(\mathrm{II}) @ \mathrm{Ag}$, affects a major lowering of the decomposition temperature (Figure 4A). The peak of cysteine at $210{ }^{\circ} \mathrm{C}$ moves and splits into peaks at $165{ }^{\circ} \mathrm{C}$ and $135{ }^{\circ} \mathrm{C}$, while retaining a small portion of the original $210{ }^{\circ} \mathrm{C}$ peak (Figure 4B). This very significant lowering reflects the known catalytic effect of silver on thermal degradation, which has been observed previously in other organically doping silver ${ }^{35,36}$ and is a direct proof of the entrapment of cysteine. The broadening of the peaks in the doped form is a reflection of a more heterogeneous environment that the dopant molecules experience, that is, of the various cage and pore geometries where the dopant molecules resides. The main gaseous products of the thermal decomposition of pure cysteine are $\mathrm{H}_{2} \mathrm{~S}$ and $\mathrm{CO}_{2}$, obtained by the cleavage of $\mathrm{C}-\mathrm{C}$ and $\mathrm{C}-\mathrm{S}$ bonds, ${ }^{37}$ are shown in Figure 4C, D. Interestingly, the presence of $\mathrm{Zn}(\mathrm{II})$ strongly influences the decomposition behavior of cysteine compared with entrapment in pure silver, indicating a strong interaction between them: firstly, the catalytic effect of silver has been attenuated in presence of zinc, as the decomposition of cysteine occurs at higher temperature; and second, there appears a peak at the elevated temperature of $550{ }^{\circ} \mathrm{C}$ (Figure 4A), which is associated with $\mathrm{CO}_{2}$, as shown by the MS-coupled to the TGA of cys/Zn(II)@Ag (Figure 4C). The absence of an $\mathrm{H}_{2} \mathrm{~S}$ signal during the calcination (Figure 4D) points to the probable forming of $\mathrm{ZnS}$ upon thermolysis. As the enthalpy of formation $\left(\Delta_{f} H_{298}^{\theta}\right)$ of $\mathrm{ZnS}(-204.6 \mathrm{~kJ} / \mathrm{mol})$ is much lower than that of $\mathrm{AgS}(-32.59$ $\mathrm{kJ} / \mathrm{mol}$ ), the formation of the thermally stable $\mathrm{ZnS}$ is preferred. Compared to the decomposition of cysteine ( 20 w.t.\% of composites), the weight loss of $\mathrm{Zn}$ (II) dehydration $(<2 \%$ w.t. $\%)$ is negligible. The strong cysteine- $\mathrm{Zn}$ interaction revealed by the TGA analysis is an important element of the WF-increase mechanism detailed in the next section. 
This TGA analysis correlates well with the WF thermal stability of cys/Zn(II)@Ag (Figure S6): It is seen that the ultrahigh WF is maintained up to 150 ${ }^{\circ} \mathrm{C}$ and decreases significantly with further heating to $200{ }^{\circ} \mathrm{C}$ and $300{ }^{\circ} \mathrm{C}$, at which the thermal decomposition of cysteine occurs according to the TGA analysis. The importance of this heating experiment is that it proves the necessary role of cysteine in the WF increase, because that increase is stable up to the point where this amino acid decomposes $\left(150^{\circ} \mathrm{C}\right)$.

To explore additionally the specific interactions of cysteine with the solid components Raman spectroscopy has been carried out. The two enhanced Raman bands in the $1500-1700 \mathrm{~cm}^{-1}$ (inset of Figure 4E) are in agreement with the reported Raman spectrum of the mercaptide $\mathrm{Zn}(\mathrm{cys}){ }_{2}{ }^{38}$ (Figure S9) which is formed in reaction (4) above. $\mathrm{Zn}$ (cys) $)_{2}$ is a component of the redox controlling "zinc-finger effect", 25,26,39 which contributes to the WF large increase. Indeed, in this context it is noteworthy to point out that the small peak at $\sim 504 \mathrm{~cm}^{-1}$ is associated with an $\mathrm{S}-\mathrm{S}$ dipeptide bond pointing to the existence of cystine (the oxidized dimer of cysteine), which again is relevant to the zinc-finger phenomenon discussed below. Notably, Shkirskiy et al ${ }^{21}$ reported that in the $\mathrm{Zn}$ oxide-hydroxide film/cysteine system, the value of the Gibbs free energy of the complex formation between these two components is $-100 \mathrm{~kJ} \mathrm{~mol}^{-1}$, that is, the formation of $\mathrm{Zn}$ (cys) $)_{2}$ is thermodynamically favored over simple adsorption, for which, $\mathrm{G}^{0}$ was about $-25 \mathrm{~kJ} \mathrm{~mol}^{-1}$. Last but not least, inelastic neutron scattering spectroscopy (INS) is also well suited to the study of the interaction of a metal with molecules, because the metal cages are essentially invisible to neutrons and the hydrogen rich organic molecules are strong scatters ${ }^{40,41}$. As shown in Figure 4F, adsorption and entrapment are totally different: In the adsorbed case, the molecule spectrum is very similar to that of pure cysteine with only minor difference, while major differences are observed in the entrapment case. It is noticeable that the disappearance of $\mathrm{S}-\mathrm{H}$ in-plane bend and significant attenuation of the $\mathrm{NH}_{3}$ rock only occurs in cys/Zn(II)@Ag, which points to the formation of Zn-cysteine complex. More Raman and INS analyses can be found in the SI (Figure S7-S10).

\section{Proposed origin of the ultra-high work function and conclusion:}

Based on the detailed multi-analytical investigations of the structure and properties of the various doped silver samples, we propose that the large increase in the work function is due to the combined effect of these components (Figure 5): Cysteine, 


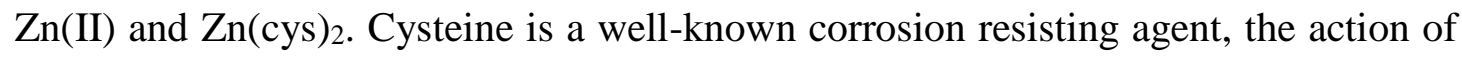
which has been attributed to the interaction of the thiol group with the metal surface. ${ }^{28,29,42}$ The adsorption of the thiol group creates a dipole barrier for charge transfer from the metal (Figure 5B), affecting an increase in the WF of the protected metal of the order of $1 \mathrm{eV}$ (Table 1). ${ }^{6,74}$ Likewise the contribution from $\mathrm{Zn}$ (II) is attributed to the well-documented surface dipole due to charge transfer at an metalinsulator interface (Figure 5B), ${ }^{44-46}$ where common insulators have been metal oxides. ${ }^{47-49}$ In this case, the suggested mechanism in the literature has been the decay of the metal wave function into the insulator where the metal conduction band overlaps with the insulator band gap. For instance, increases of the order of $0.5-1 \mathrm{eV}$ have been observed with titania and silica. ${ }^{49}$ Indeed, we observed increases of 0.1-1.0 eV when silver was doped with zinc but without cysteine (Table 1). The second shell scattering of Zn EXAFS and the XRD, indicate that the extremely small Zn(II) is homogeneously distributed and this facilitates the efficient formation of a metal-dielectric interface between $\mathrm{Zn}(\mathrm{II})$ and the silver matrix. The metal-insulator distance is also a key aspect in determining the work function change. Pacchioni et. al. ${ }^{49}$ showed that efficient WF changes occur at a distance range of 0.72-2.73 $\AA$. In the cys/Zn(II)@Ag, the Ag-O distance at $\mathrm{Zn}(\mathrm{II}) / \mathrm{Ag}$ interface can be estimated to be $\sim 1.66 \AA$ (Figure S4C, detailed estimation has been discussed in $\mathbf{S I}$ ), which is therefore supposed to be an appropriate range for efficient charge transfer.

The third contributor to the WF increase is the combination of the two dopants, cysteine and $\mathrm{Zn}(\mathrm{II})$, namely the mercaptide $\mathrm{Zn}(\mathrm{cys})_{2}$ (eq. 4), which is a component of the cysteine- $\mathrm{Zn}^{2+}$ (“zinc-finger”) electron-trap redox mechanism. This mechanism, commonly found in in biochemical systems, acts as a powerful anti-oxidation agent by shuttling electrons back and forth between two Zn/cysteine species, ${ }^{25,26,39}$ (Figure 5B). Thus, cys-S-Zn-S-cys contributes to the blocking of the ability of the metal electron to leave it (increase in WF) by acting as an electron releasing source, while the second component of the zinc finger - the cys-S-S-cys $/ \mathrm{Zn}^{2+}$ pair - can act as an electron trap for an injected electron; both $\mathrm{Zn}(\mathrm{cys})_{2}$ and cystine were indeed detected, as described above. Also supporting our observations and conclusions are the computational studies cited in the Introduction, where an ultrahigh WF increase $(\sim 4 \mathrm{eV})$ of silver has been predicted in a triple organic/silicone/silver system, ${ }^{9}$ quite close to the ultrahigh WF increase $(>3 \mathrm{eV})$ we achieved in our system. We also note that recent investigations reveal that the WF alternation by chemical modification is highly dependent on the 
molecules orientation (bending and rotation of substituents) $)^{8,10,50}$ at the metal/dielectric interface. And indeed, as revealed by the INS spectra, the molecular state of cysteine in the entrapped case has been changed significantly.

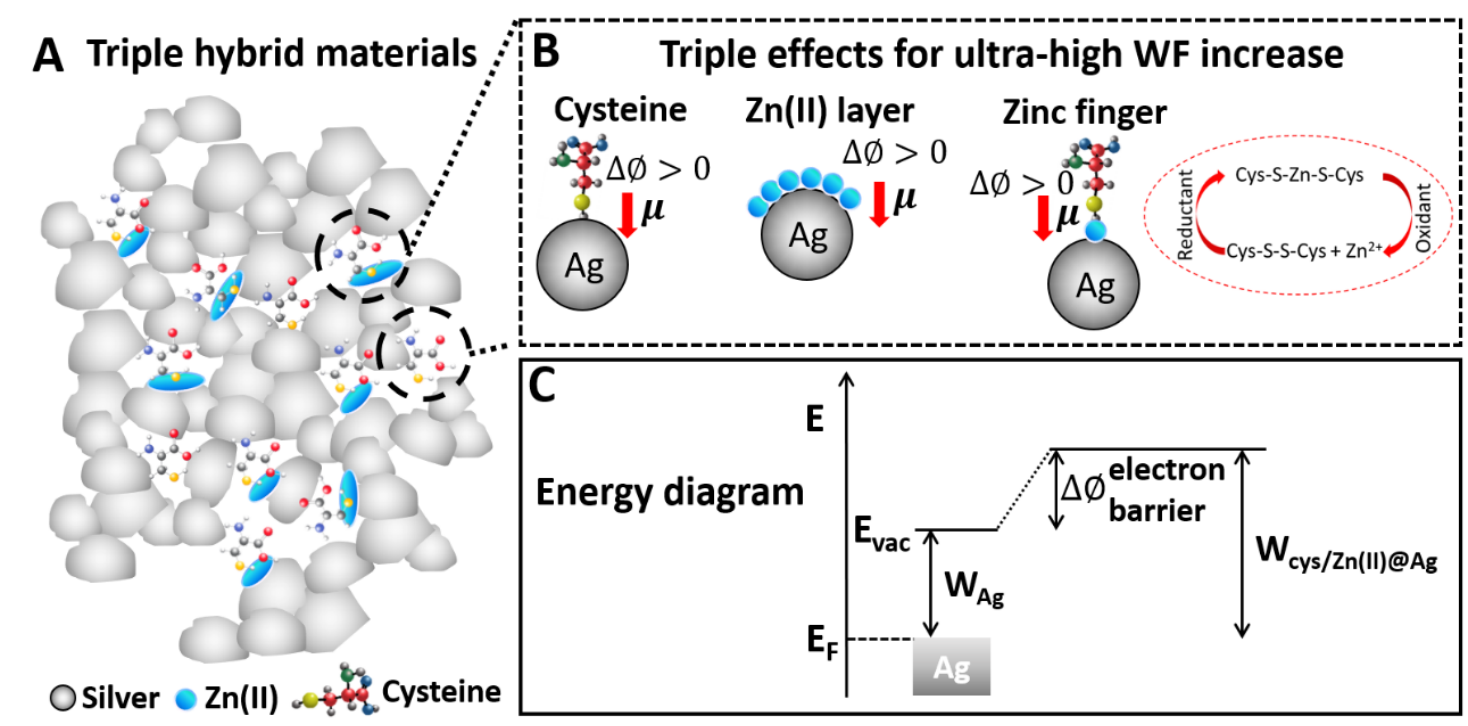

Figure 5. The proposed mechanism of the ultrahigh work function effect. A) The general structure of the doped silver. B) The origin of the of the ultra-high increase in the WF: The surface dipole/charge transfer is affected by three components: The interfaces of cysteine/Ag (left) and of $\mathrm{Zn}(\mathrm{OH})_{2} / \mathrm{Ag}$ (center) and the cysteine- $\mathrm{Zn}^{2+}$ ("zinc-finger")/Ag interface (right). C) The energy diagram: The increase in the WF is due to the electron barrier, keeping the Fermi level of Ag unaffected.

\section{Conclusion}

Here we report affecting an ultrahigh WF of silver, reaching a value of $7.42 \mathrm{eV}$, which, to the best of our knowledge is the highest recorded for a metal by a chemical modification, and agrees with computational predictions. The approach is new, and involves $3 \mathrm{D}$ doping of the bulk of the metal, rather than the common $2 \mathrm{D}$ surface treatment. Co-doping with the L-cysteine and hydrolyzed zinc (II) leads to contributions to the increase in the WF by each component separately by affecting directly the charge transfer ability of the metal, and synergistically by the combination of the two components through the known Zn-Cys finger effect. The ability to tailor WF changes from the standard value of silver and up over a wide range, will certainly find its applications wherever tuning of the WF is needed for the design of charge transport devices. For example, silver has been applied as a hole-extraction material in inverted polymer solar cells (IPSCs) owing to its electronic properties and its work 
function. In an IPSC, the WF should be sufficiently high to allow for the build-up of the electric field within the active component in the cell to maximize the extraction of holes from the active material. ${ }^{9,51}$

\section{Experimental Section}

Experimental details of the chemicals, synthesis details and materials characterization are provided in the Supporting Information.

Acknowledgments: Supported by the Ministry of Science, Technology \& Space of Israel through grant No. 3-12948. The authors acknowledge the Diamond Light Source, UK (project no. SP18835, B18 beamline) and the ISIS facility, UK (project no. 1720114, TOSCA spectrometer) for the provision of beamtime. J. H. and B. M. acknowledge

Newton Funding for supporting to use the ISIS facility. We thank Mr. Giannantonio Cibin for assistance in performing the XAFS measurements.

\section{References:}

[1] N. D. Lang, W. Kohn, Phys. Rev. B 1971, 3, 1215.

[2] I. H. Campbell, S. Rubin, T. A. Zawodzinski, J. D. Kress, R. L. Martin, D. L. Smith, N. N. Barashkov, J. P. Ferraris, Phys. Rev. B 1996, 54, R14321

[3] H. Zhang, J. Tang, Q. Zhang, G. Zhao, G. Yang, J. Zhang, O. Zhou, L. Qin, Adv. Mater. 2006, 18, 87-91

[4] J. P. Barnak, R. S. Chau, C. Liang, US Patent 2006, US7022559B2.

[5] B. de Boer, A. Hadipour, M. M. Mandoc, T. van Woudenbergh, P. W. M. Blom, Adv. Mater. 2005, 17, 621-625.

[6] M. L. Sushko, A. L. Shluger, Adv. Mater. 2009, 21, 1111-1114.

[7] V. B. Engelkes, J. M. Beebe, C. D. Frisbie, J. Am. Chem. Soc. 2004, 126, 14287-14296.

[8] O. T. Hofmann, D. A. Egger, E. Zojer, Nano Lett. 2010, 10, 4369-4374.

[9] P. D. Taylor, D. A. Osborne, S. A. Tawfik, T. Morishita, M. J. S. Spencer, Phys. Chem. Chem. Phys. 2019, 21, 7165-7173.

[10] O. T. Hofmann, H. Glowatzki, C. Bürker, G. M. Rangger, B. Bröker, J. Niederhausen, T. Hosokai, I. Salzmann, R.-P. Blum, R. Rieger, J. Phys. Chem. C. 2017, 121, 2465724668. 
[11] J. He, L. lagher, L. Etgar, D. Avnir, Chem. Commun. 2018, 54, 7203-7206.

[12] D. Avnir, Adv. Mater. 2018, 30, 1706804 .

[13] D. Avnir, Acc. Chem. Res. 2013, 47, 579-592

[14] N. Ralbag, M. Mann-Lahav, E. S. Davydova, U. Ash, R. Galed, M. Handl, R. Hiesgen, E. Magliocca, W. Mustain, J. He, P. Cong, A. M. Beale, G. S. Grader, D. Avnir, D. R. Dekel, Matter 2019, 1, 959-975.

[15] T. S. Bauer, B. Menagen, D. Avnir, Z. Hayouka, Sci. Rep. 2019, 9, 1-8

[16] B. Menagen, D. Avnir, ACS Biomater. Sci. Eng. 2019, 5, 2355-2364.

[17] L. Shapiro, D. Avnir, ChemCatChem 2017, 9, 816-823.

[18] N. Ralbag, I. Felner, D. Avnir, Phys. Rev. B. 2019, 99, 64411.

[19] R. Ben-Knaz, D. Avnir, Biomaterials. 2009, 30, 1263-1267.

[20] P. Li, Z. P. Xu, M. A. Hampton, D. T. Vu, L. Huang, V. Rudolph, A. V. Nguyen, A. V. J. Phys. Chem. C. 2012, 116, 10325-10332

[21] V. Shkirskiy, P. Keil, H. Hintze-Bruening, F. Leroux, F. Brisset, K. Ogle, P. Volovitch, Corros. Sci. 2015, 100, 101-112.

[22] N.Pace, E. Weerapana, Biomolecules 2014, 4, 419-434.

[23] F. D. Urnov, J. C. Miller, Y.-L. Lee, C. M. Beausejour, J. M. Rock, S. Augustus, A. C. Jamieson, M. H. Porteus, P. D. Gregory, M. C. Holmes, Nature 2005, 435, 646.

[24] M Bibikova, K Beumer, JK Trautman, D Carroll, Science 2003, 300, 764.

[25] W. Maret, Antioxid. Redox Signal. 2006, 8, 1419-1441.

[26] K.-D. Kröncke, L.-O. Klotz, Antioxid. Redox. Signal. 2009, 11, 1015-1027.

[27] B. O. Leung, F. Jalilehvand, V. Mah, M. Parvez, Q. Wu, Inorg. Chem. 2013, 52, 45934602.

[28] N. H. Helal, W. A. Badawy, Electrochim. Acta. 2011, 56, 6581-6587

[29] M. B. Radovanović, M. B. Petrović, A. T. Simonović, S. M. Milić, M. M. Antonijević, Environ. Sci. Pollut. Res. 2013, 20, 4370-4381.

[30] D. Kesavan, M. Gopiraman, N. Sulochana, Chem. Sci. Rev. Lett. 2012, 1, 1-8.

[31] S. Fischer, A. C. Papageorgiou, M. Marschall, J. Reichert, K. Diller, F. Klappenberger, F. Allegretti, A. Nefedov, C. Wöll, J. V. Barth, J. Phys. Chem. C. 2012, 116, 20356-20362.

[32] Q. Chen, N. V. Richardson, Nat. Mater. 2003, 2, 324.

[33] T. N. Ramesh, T. L. Madhu, Int. J. Inorg. Chem. 2015, 536470. 
[34] A. K. Yadav, S. M. Haque, S. Tripathi, D. Shukla, M. A. Ahmed, D. M. Phase, S. Bandyopadhyay, S. N. Jha, D. Bhattacharyya, RSC Adv. 2016, 6, 74982-74990

[35] H. Behar-Levy, G. E. Shter, G. S. Grader, D. Avnir, Chem. Mater. 2004, 16, 31973202.

[36] G. Nesher, M. Aylien, G. Sandaki, D. Avnir, G. Marom, Adv. Funct. Mater. 2009, 19, 1293-1298.

[37] V. A. Yablokov, Y. A. Vasina, I. A. Zelyaev, S. V. Mitrofanova, Russ. J. Gen. Chem. 2009, 79, 1141.

[38] S. Foley, M. Enescu, Vib. Spectrosc. 2007, 44, 256-265.

[39] X. Wu, N. H. Bishopric, D. J. Discher, B. J. Murphy, K. A. Webster, Mol. Cell. Biol. 1996, 16, 1035-1046.

[40] S. F. Parker, A. J. Ramirez-Cuesta, L. Daemen, Spectrochim. Acta Part A Mol. Biomol. Spectrosc. 2018, 190, 518-523.

[41] S. F. Parker, F. Fernandez-Alonso, A. J. Ramirez-Cuesta, J. Tomkinson, S. Rudic, R. S. Pinna, G. Gorini, J. F. Castañon, J. Phys. Conf. Ser. 2014, 12003.

[42] K. M. Ismail, Electrochim. Acta 2007, 52, 7811-7819.

[43] R. W. Zehner, B. F. Parsons, R. P. Hsung, L. R. Sita, Langmuir 1999, 15, 1121-1127.

[44] S. Prada, U. Martinez, G. Pacchioni, Phys. Rev. B 2008, 78, 235423.

[45] S. Choi, D.-H. Lee, S. G. Louie, J. Clarke, Phys. Rev. Lett. 2009, 103, 197001.

[46] C. Cen, S. Thiel, G. Hammerl, C. W. Schneider, K. E. Andersen, C. S. Hellberg, J. Mannhart, J. Levy, Nat. Mater. 2008, 7, 298.

[47] M. Sterrer, T. Risse, U. M. Pozzoni, L. Giordano, M. Heyde, H.-P. Rust, G. Pacchioni, H.-J. Freund, Phys. Rev. Lett. 2007, 98, 96107.

[48] S. Lu, Z. Qin, Q. Guo, G. Cao, Appl. Surf. Sci. 2017, 392, 849-853.

[49] L. Giordano, F. Cinquini, G. Pacchioni, Phys. Rev. B 2006, 73, 45414.

[50] E. Zojer, T. C. Taucher, O. T. Hofmann, Adv. Mater. Interfaces 2019, 190058

[51] C. Hu, D. Liu, Y. Xiao, L. Dai, Prog. Nat. Sci. Mater. Int. 2018, 28, 121-132. 


\section{Entry for the Table of Contents:}

An ultra-high increase in the WF of silver, from 4.26 to $7.42 \mathrm{eV}$, by the 3D dualentrapment of WF modifying components - L-cysteine and $\mathrm{Zn}(\mathrm{OH})_{2}$ - within the metal. The WF enhancement mechanism which is based on affecting directly the charge transfer ability of the metal separately by cysteine and hydrolyzed zinc(II), and synergistically by the combination of the two components through the known $\mathrm{Zn}$ cysteine finger redox trap effect.
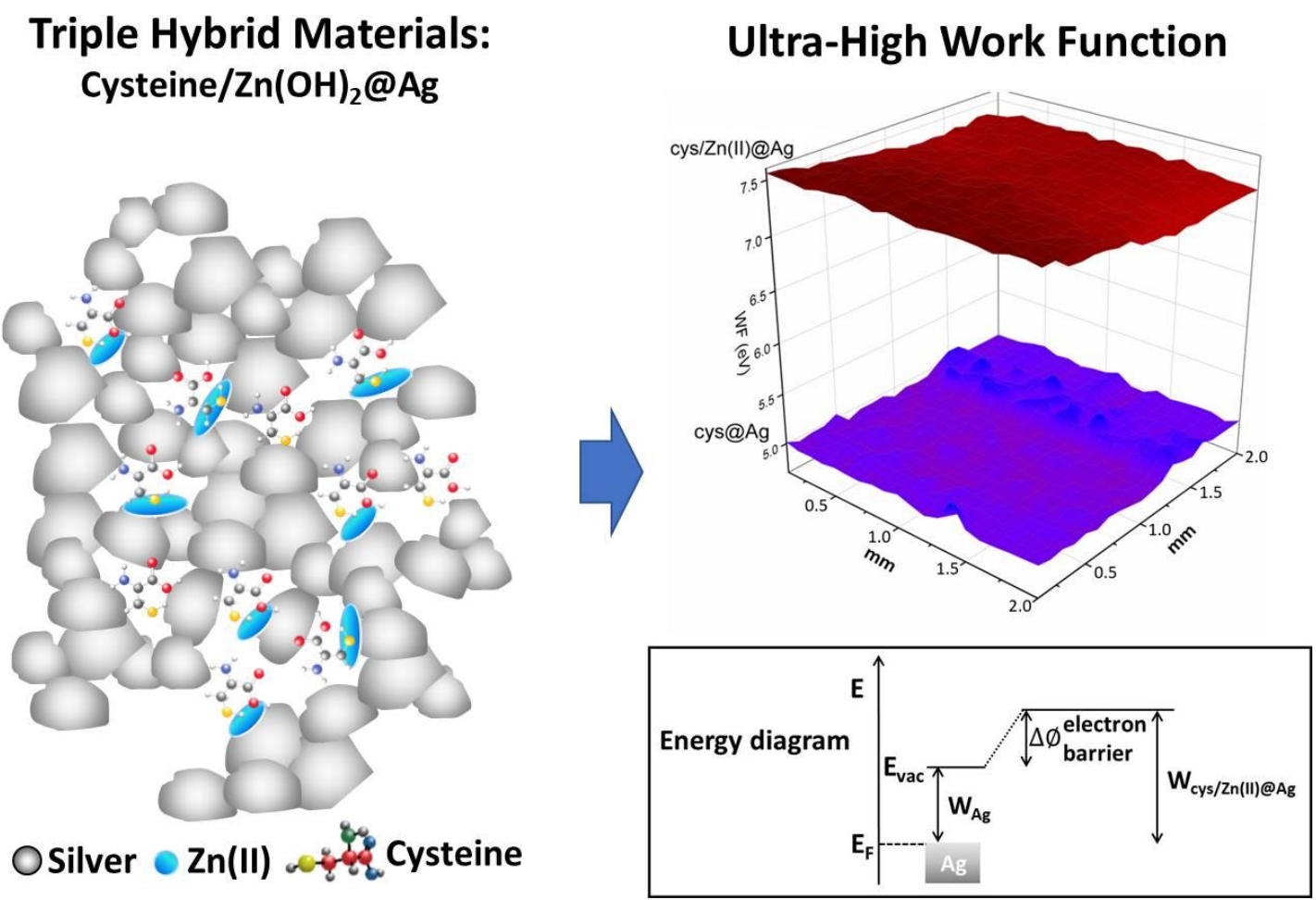

J. He, J. Armstrong, P. Cong, B. Menagen, L. Igaher, A. M. Beale, L. Etgar, D. Avnir Page No. - Page No.

A Triple Hybrid Silver Material Demonstrates an Ultra-High Work Function. 